Proceedings - 19th International Conference - IEEE/EMBS Oct. 30 - Nov. 2, 1997 Chicago, IL. USA

\title{
MICROFABRICATED DEVICE FOR ARTERIAL WALL AND ATHEROSCLEROTIC PLAQUE PENETRATION
}

\author{
James R. Kneller*, Clarence C. Wu, Simon Watkins, Ahmed Nadeem, Michael Reed ${ }^{\mathfrak{I}}$, Lee Weiss ${ }^{\mathfrak{I}}$, \\ Marc D. Feldman, ${ }^{\Im}$ Carnegie Mellon University, Hamerschlag Hall, Pittsburgh, PA 15213 USA \\ Division of Cardiology, School of Medicine, University of Pittsburgh, F-392 PUH, \\ 200 Lothrop St., Pittsburgh, PA 15213 USA (*email: knelle@card2.cath.upmc.edu)
}

\begin{abstract}
Percutaneous coronary intervention is currently used to treat coronary atherosclerosis but is plagued by restenosis. The atherosclerotic plaque acts as a barrier, preventing the delivery of drugs or gene therapy to prevent restenosis. We hypothesize that microfabricated probes, manufactured using silicon fabrication technology, can penetrate through atherosclerotic plaque, creating paths for therapeutic delivery. Two sets of microfabricated probes $(65 \pm 15$ and $140 \pm 20 \mu \mathrm{m}$ ) were deployed in normal and atherosclerotic rabbit iliac artery segments $(n=5$ each) under distention pressures of 100, 200, 300, and 500 mmHg, to simulate deployed stents. The tissues were fixed while the probes remained in place and analyzed using standard SEM, TEM and light microscopy techniques to evaluate the extent and nature of vessel penetration. In healthy tissue, microprobes are able to pierce the internal elastic lamina and penetrate the media, with the highest probes nearly reaching the media/adventitia boundary. Atherosclerotic plaque is pierced by microprobes at all intraluminal pressures examined. These results indicate that microprobes may serve as a technique to penetrate the atherosclerotic plaque for the purpose of delivering therapeutics beyond the plaque.
\end{abstract}

Index Terms-Microprobes, microelectromechanical systems, plaque, restenosis, intracoronary stent.

\section{INTRODUCTION}

Coronary atherosclerosis is the leading cause of death. Percutaneous Transluminal Coronary Angioplasty (PTCA), or "balloon angioplasty" is currently used to treat stenoses but is plagued by abnormal vascular wound healing and remodeling [1-3] which leads to restenosis. Although the initial success of balloon angioplasty is over $95 \%$, one-third of patients return within six months due to restenosis, or the reoccurrence of the blockage. Intracoronary stenting has been shown to effectively reduce the incidence of restenosis ( $32 \%$ vs. $42 \%, p<0.05$ ) [4]. In addition, stents offer a platform for drug or gene therapy to treat the diseased artery. However, no stent design offers a solution on how to penetrate the diffuse atherosclerotic barrier so therapeutics can reach the portions of the vessel where wound healing and remodeling reside.

Microfabrication is an emerging engineering technology. Using tools and batch fabrication processes developed for the integrated circuit industry, complex electromechanical systems (MEMS) can be achieved. Applications of MEMS technology has not been limited to traditional engineering disciplines, but also includes numerous biological applications [5-8]. Micro-piercing structures have been used to transfect tobacco leaves and nematodes by creating a path for genetic material to enter the cell [5-6]. Furthermore, sharp micromechanical probes have been fabricated for bonding biologic tissues such as blood vessels which may have the potential for local drug delivery [9]. Fabrication of a coronary stent based on MEMS technology has also been demonstrated recently [10]. The integration of various microfabrication technologies may provide a solution for a cost-effective local delivery system which incorporates the advantage of stenting while achieving therapeutic delivery beyond the atherosclerotic plaque.

Accordingly, we hypothesize that microprobes can produce controlled incisions into the arterial wall and pierce atherosclerotic plaque. In this way, microprobes integrated on the surface of an intracoronary stent may facilitate the delivery of drug or gene therapy through atherosclerotic plaque.

\section{METHODS}

\section{A. Microprobe Fabrication}

The microprobes are made from silicon, and are manufactured using silicon fabrication technology. Manufacturing processes include photolithography and anisotropic wet etching, where a $1.5 \mu \mathrm{m} \mathrm{SiO}{ }_{2}$ layer is grown at $1000{ }^{\circ} \mathrm{C}$ in wet oxygen on (100) oriented silicon wafers, forming a mask pattern [11]. Mask formation is followed by 
two anisotropic etching steps which first create a truncated pyramid bounded by $\{411\}$ planes followed by further undercutting etching to complete the pyramid under the oxide mask [12].

\section{B. Simulating stent deployment}

When applying the microprobes, post-stenting vessel distention was simulated. Clinically, stent deployment results in 10 to $30 \%$ vessel distention, corresponding to the maximum distensibility of the elastin fibers in the vessel wall [13]. This distention is reproduced in a harvested vessel which has relaxed both circumferentially and longitudinally by stretching the vessel 10 to $30 \%$ beyond its in vivo circumference $(\sim 1.4$ times its relaxed circumference) [14]. The intraluminal pressure seen by the lumen during stent deployment depends on the stiffness of the tissue, which increases with atherosclerotic plaque formation and the progression from a cellular to calcified lesion. The intraluminal pressure range $(\Delta P)$ needed to distend healthy and diseased rabbit tissues was calculated from vessel wall compliance $(C)$ and volume change $(\Delta V)$ measurements.

$$
C=\frac{\Delta V}{\Delta P}
$$

The compliance of atherosclerotic rabbit vessels with 10 and $30 \%$ distention were $0.058 \cdot 10^{-3} \mathrm{ml} / \mathrm{mmHg}$ and $0.061 \cdot 10^{-3}$ $\mathrm{ml} / \mathrm{mmHg}$ respectively [15], corresponding to a pressure range of 150 to $500 \mathrm{mmHg}$. Based on these figures the microprobes were applied at 100,200,300, and $500 \mathrm{mmHg}$.

\section{Animal Model of Atherosclerosis}

The iliac arteries from $(n=10)$ New Zealand White rabbits were used to evaluate two available microprobe geometries: 1) $65 \pm 15 \mu \mathrm{m}$ and 2) $140 \pm 20 \mu \mathrm{m}$ microprobes. The atherosclerotic model was created by feeding $(n=5)$ animals a hypercholesterolemic diet containing $5 \%$ cholesterol and $7 \%$ peanut oil for a period of three weeks. At the conclusion of the three week period, arterial injury was performed by balloon injury followed by an additional three weeks of the high cholesterol diet. This method has been shown to reproducibly create stenoses that resemble human atherosclerotic lesions.

\section{Tissue Processing}

Both iliac arteries were simultaneously dissected from the distal side of the inguinal ligament to the bottom of the aorta. The base of the aorta was ligated to allow cannulation of the iliac artery, permitting a brief pressure perfusion with a medium to rinse the vessel free of blood. The vessel was then dissected free of excess retroperitoneal tissue and opened longitudinally along its dorsal aspect. It was then clamped as a planar sheet at its in vivo length to prevent collapse or buckling of the segment according to the method of Fry et al. [16]. The vessels were sectioned and imaged using standard structural biology tissue processing and imaging techniques for scanning electron microscopy (SEM), transmitted electron microscopy (TEM), and light microscopy.

\section{Experiment Apparatus and Tissue Imaging}

The apparatus shown in Fig. 1 was designed to apply the microprobes to the tissue. Harvested vessel segments were mounted, endothelial side up, on the tissue holding device. The device is 10 to $30 \%$ wider than the mean in vivo vessel circumference [17]. During microprobe application the chamber was purged with $2.5 \%$ gluteraldehyde for 20 minutes, preserving the interaction between the tissue and the microprobes. Following tissue fixation, the tissue damaged by the device pins adjacent to the chamber walls was trimmed from the specimen. Separate tissue processing protocols were then followed for imaging the tissue/microprobe interaction using SEM, TEM, or light microscopy.

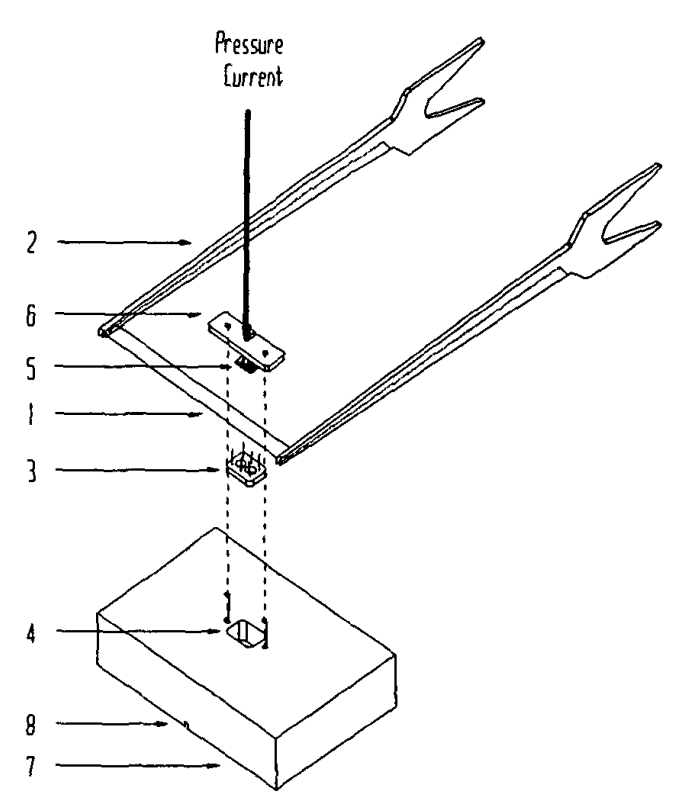

Fig. 1: The filleted artery (1) is clamped (2) at its in vivo length. A segment of tissue is mounted luminal side up on the device (3) and stretched longitudinally and circumferentially. The mounted tissue is held by the device (4) as pressure is applied to the microprobes (5) by a plunger (6). 


\section{RESULTS}

Microfabricated probes were found to produce uniform, controlled incisions into the arterial wall without damaging the endothelium adjacent to the microprobes (Fig. 2).
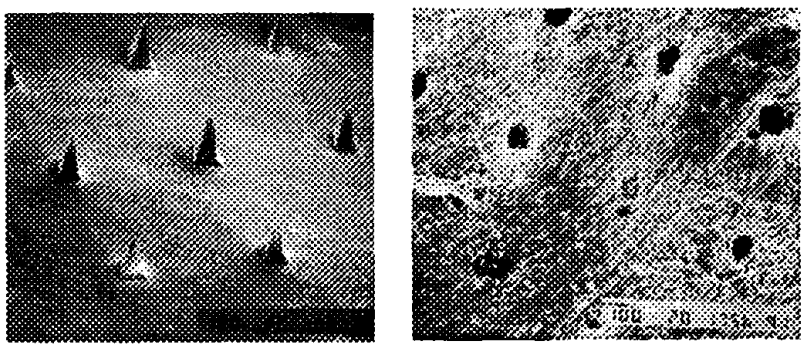

Fig. 2: $140 \pm 20 \mu \mathrm{m}$ microprobes (left) on a planar substrate, and the corresponding tissue incisions (right) into a distended arterial segment when applied at a pressure of $200 \mathrm{mmHg}$. The microprobes make controlled incisions into the vessel wall without damaging the adjacent endothelium.

$65 \pm 15 \mu \mathrm{m}$ microprobes failed to pierce the internal elastic lamina at all applied pressures while $140 \pm 20 \mu \mathrm{m}$ microprobes pierced the internal elastic lamina at all applied pressures in normal rabbit iliac arteries (Fig. 3). The microprobes were found to pierce through the internal elastic lamina (IEL) and then cut into and compress the smooth muscle cells. The depth of penetration was proportional to the applied pressure. In no cases did the microprobes reach the external elastic lamina (EEL).

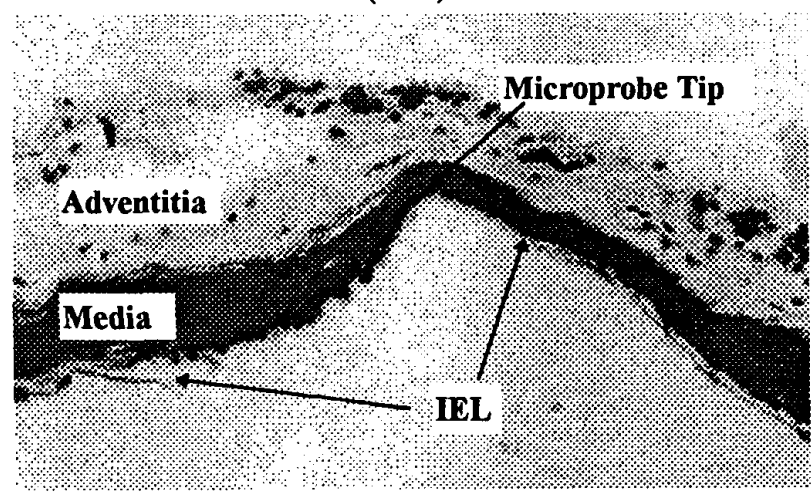

Fig. 3: $140 \pm 20 \mu \mathrm{m}$ microprobe penetration into a healthy rabbit iliac artery at $200 \mathrm{mmHg}$. With this pressure, the microprobes cut through the IEL and into the media.

The rabbit atherosclerotic lesions were composed of smooth muscle cells and foam cells on the luminal side of the plaque. They were cut and compressed at the tips of the microprobes when applied at the pressure range examined (150 to $500 \mathrm{mmHg}$ ). The microprobes were found to penetrate through the plaque. However, they could not penetrate through plaque thicker than the microprobe height (Fig. 4).

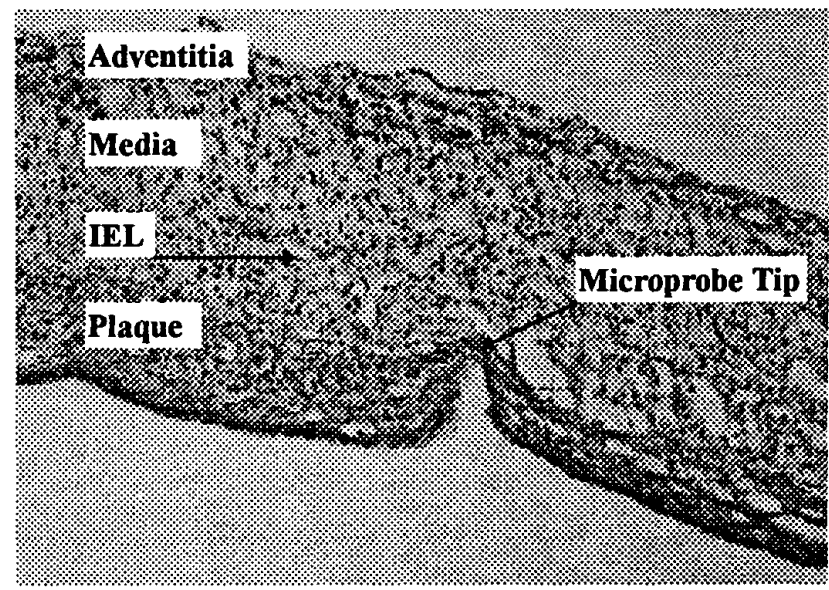

Fig. 4: $140 \pm 20 \mu \mathrm{m}$ microprobe penetration into a rabbit atherosclerotic lesion at $100 \mathrm{mmHg}$. At this pressure, the microprobes cut into the plaque.

\section{DISCUSSION}

Microfabricated probes, created using MEMS manufacturing techniques, can function as sharp piercing devices. Microprobes are able to pierce the arterial IEL of normal vessels and penetrate into atherosclerotic plaque. The damage made by these incisions is controlled and does not affect the adjacent tissue, demonstrating the ability of this device to minimize damage.

The $65 \pm 15 \mu \mathrm{m}$ microprobes were unable to pierce the IEL of healthy vessels, while $140 \pm 20 \mu \mathrm{m}$ microprobes were able to penetrate the same vessels at all intraluminal pressures examined. This result indicates that microprobe height is the parameter controlling whether or not the microprobe tips reach the media. Furthermore, $140 \pm 20 \mu \mathrm{m}$ microprobes were not tall enough to reach the EEL, indicating that microprobes of certain height safely penetrate the media without transecting the entire vessel.

The rabbit model of atherosclerosis resulted in classic plaque formation, composed - of migrated medial smooth muscle cells, aggregate foam cells, and a disrupted IEL [18]. $65 \pm 15 \mu \mathrm{m}$ and $140 \pm 20 \mu \mathrm{m}$ cut into plaque at all stent deployment intraluminal pressures. This result indicates that taller microprobes need to be developed for complete atherosclerotic plaque penetration.

\section{CONCLUSIONS}

The emerging field of microfabrication has given rise to devices with a variety of bioapplications. The ability of the microprobe to penetrate arterial walls and atherosclerotic plaque has been demonstrated. Microprobes of sufficient 
height are able to produce controlled incisions into muscular arteries and penetrate atherosclerotic plaque at all intraluminal post-stenting pressures. These results indicate that microprobes of sufficient height may reliably penetrate through the intima and facilitate the delivery of therapeutics through the atherosclerotic plaque. Hence, microprobes incorporated on the surface of an intravascular stent may possibly be used to deliver drug or gene therapy to the diseased vessel. Such a stent would underscore the potential for MEMS devices to treat human diseases.

\section{REFERENCES}

[1] Ip JH, Fuster V, Israel D, "The role of platelet thrombin and hyperplasia in restenosis after coronary angioplasty," J Am Coll Cardiol 1991; 17: 77B-88B.

[2] Forrester J, Fishbein M, Helfant R, "A paradigm of restenosis based on cell biology: clues for the development of new preventive therapies," J Am Coll Cardiol 1991;17: 77B-88B.

[3] Katuta T, Currier JW, Haudenschild CC, Ryan TJ, Faxon DP, "Differences in compensatory vessel enlargement, not neointima formation, account for restenosis following angioplasty in the hypercholesterolemic rabbit model," Circulation 1994; 89: 2809-2815.

[4] Fischman DL, Leon MB, Baim DS, et al, "A randomized comparison of coronary stent placement and balloon angioplasty in the treatment of coronary artery disease. Stent Restenosis Study Investigators," N Engl J 1995; 16: 437-440.

[5] Hashmi S, Ling P, Hashmi G, Reed M, et al: "Genetic transformation of nematodes using arrays of micromechanical piercing structures," BioTechniques 1995; 19: 766-770.

[6] Trimmer W, Ling P, Chin CK, Orton P, Gaugler R, Hashmi G, Hashmi S, Brunett B, Reed ML, "Injection of DNA into plant and animal tissues with micromechanical piercing structures," Proceedings of the Eighth International Workshop on Micro Electro Mechanical Systems (MEMS-95) 1995; 111-115.

[7] Reed ML, Han H, Weiss LE, "Silicon micro-velcro," Advanced Materials 1992; 4: 48-51.

[8] Han H, Weiss LE, Reed ML, "Mating and piercing micromechanical structures for surface bonding applications," IEEE Workshop Micro Electro Mech Syst (MEMS-91) 1991; 253-258.

[9] Dizon R, Han H, Russell AG, Reed ML, “An ion milling pattern transfer technique for fabrication of three-dimensional micromechanical structures," $J$ of MicroElectroMechan Syst 1993; 2(4): 151-159.

[10] Amon M, Winkler S, Dekker A, Bolz A, et al, "Introduction of a new coronary stent with enhanced radioopacity and hemocompatibility," Proceedings IEEE Engr in Med and Bio 1995 (CD Version).

[11] Dizon R, Han H, Reed ML, "Single-mask processing of micromechanical piercing structures using ion milling," IEEE Transactions 1993; 48-52.

[12] Wu X, Wen $\mathrm{KH}$, "Compensating corner undercutting in anisotropic etching of (100) silicon," Sensors and Actuators 1989; 18, 207-215.

[13] Stark L, Aganwal G: Biomaterials, Plenum Press, New York: 1969.

[14] Fry DL, Cornhill FJ, Sharma H, et al., "Uptake of low density lipoprotein, albumin, and water by deendothelialized in vitro minipig aorta," Arteriosclerosis September/October 1986; 6: 475-490.

[15] Zuckerman et al, "Arterial hemodynamics in a rabbit model of atherosclerosis," Sept 1989; Am J Physiol 257: H891-H897.

[16] Fry DL, Mahley RW, Suk YO, "Effect of arterial stretch on transmural albumin and Evan's blue dye transport," Am J Physiol 240: H645-H649, 1981.

[17] Burcelli SA, "Hemodynamics and atherogenesis: low density lipoprotein metabolism and endothelial cell morphology," PhD Thesis, University of Pittsburgh School of Engineering, 1990.

[18] Muller DW, Ellis SG, Topol EJ, "Experimental Models of Coronary Artery Restenosis," J Am Coll Cardiol 1992; 19: 418-32. 